\title{
Point prevalence survey of antimicrobial use and healthcare-associated infections in Belgian acute care hospitals: results of the Global-PPS and ECDC-PPS 2017
}

Eline Vandael ${ }^{1 *}$ D , Katrien Latour ${ }^{1}$, Herman Goossens ${ }^{2}$, Koen Magerman $^{3,4}$, Nico Drapier ${ }^{2}$, Boudewijn Catry ${ }^{1,5}$, Ann Versporten ${ }^{2,3}$ and the Belgian Point Prevalence Survey Study Group

\begin{abstract}
Background: The point prevalence survey of healthcare-associated infections (HAls) and antimicrobial use organized by the European Centre for Disease Prevention and Control (ECDC-PPS) and the Global Point Prevalence Survey of antimicrobial consumption (Global-PPS) were simultaneously performed in Belgian acute care hospitals in 2017.

Methods: Belgian acute care hospitals were invited to participate in either the ECDC or Global-PPS. Hospital/ward/ patient-level data were collected between September-December 2017. All patients present in the wards at 8 a.m. on the day of the PPS were included. The data of the ECDC and Global-PPS on antimicrobial consumption were pooled. Detailed data on HAls were analysed for ECDC-PPS.

Results: Overall, 110 Belgian acute care hospital sites participated in the ECDC and Global-PPS (countrywide participation rate: $81.4 \%, 28,007$ patients). Overall, a crude prevalence of patients with at least one antimicrobial of $27.1 \%$ (95\% confidence interval (Cl) 26.5-27.6\%) was found. The most frequently reported indications were pneumonia (23.2\%), urinary tract infections (15.2\%) and skin and soft tissue infections (11.9\%). The reason for antimicrobial use was recorded for $81.9 \%$ of the prescriptions, a stop/review date for $40.8 \%$ and compliance with local antibiotic guidelines for $76.6 \%$. In the ECDC-PPS, the crude prevalence of patients with at least one HAl was $7.3 \%(95 \% \mathrm{Cl} 6.8-7.7 \%)$. Most frequently reported HAls were pneumonia (21.6\%) and urinary tract infections (21.3\%).
\end{abstract}

Conclusions: HAl and antimicrobial use prevalence remained stable in comparison with the previous PPS (7.1\% and 27.4\% in 2011 and 2015, respectively). Belgian hospitals should be further stimulated to set local targets to improve antibiotic prescribing and reduce HAl.

Keywords: Point prevalence survey, Antimicrobial consumption, Healthcare-associated infections, Belgium, Acute care hospitals

\footnotetext{
* Correspondence: Eline.Vandael@sciensano.be

${ }^{1}$ Healthcare-associated infections and antimicrobial resistance, Sciensano, Rue

Juliette Wytsmanstraat 14, 1050 Brussels, Belgium

Full list of author information is available at the end of the article
}

(c) The Author(s). 2020 Open Access This article is distributed under the terms of the Creative Commons Attribution 4.0 International License (http://creativecommons.org/licenses/by/4.0/), which permits unrestricted use, distribution, and reproduction in any medium, provided you give appropriate credit to the original author(s) and the source, provide a link to the Creative Commons license, and indicate if changes were made. The Creative Commons Public Domain Dedication waiver (http://creativecommons.org/publicdomain/zero/1.0/) applies to the data made available in this article, unless otherwise stated. 


\section{Introduction}

Healthcare-associated infections (HAIs) and antimicrobial resistance (AMR) are well-known threats in healthcare. Point prevalence studies (PPS) have a wellestablished methodology to measure the prevalence of antimicrobial use and HAIs in hospitals. Results of PPS can be used to evaluate quality indicators, to follow-up antimicrobial stewardship and infection control programs, and to support decision-making [1].

In 2007, a first countrywide PPS of HAIs was conducted in 63 Belgian acute care hospitals. The prevalence of patients with at least one HAI was $6.0 \%$. The most common infections were urinary tract infections (UTIs; 23.9\%), lower respiratory tract infections (RTIs; 20.1\%) and surgical site infections (SSIs; 14.6\%) [2].

In 2011, the European Centre for Disease Prevention and Control (ECDC) organized a first European PPS on HAIs and antimicrobial use in European acute care hospitals. The survey's objectives were to estimate the total burden of HAIs and antimicrobial use in acute care hospitals in the European Union (EU) and therefore provided a standardized tool for hospitals to identify targets for quality improvement [3]. The EU prevalence of patients with at least one HAI was 6.0\% (country range 2.3-10.8\%). In the participating Belgian acute care hospitals $(N=52$ sites), this prevalence of patients with HAIs was $7.1 \%$ (95\% confidence interval (95\% CI): 6.1-8.3\%). The EU prevalence of patients receiving at least one antimicrobial agent was $35.0 \%$ (country range 21.4$54.7 \%$ ). In Belgium, a prevalence of antimicrobial use of 28.9\% (95\% CI: 26.8-31.1\%) was reported [3]. In 20162017, a second European PPS was organized by ECDC.

Besides the ECDC-PPS, the Global Point Prevalence Survey of Antimicrobial Consumption and Resistance (Global-PPS) is organized by the University of Antwerp to monitor the ratios of antimicrobial prescribing and resistance in hospitalized inpatients on a worldwide level, with special attention to low-middle income countries. The first Global-PPS was performed in 2015. Worldwide, $34.4 \%$ of the included patients received at least one antimicrobial agent [4]. In the included Belgian acute care hospitals ( $N=100$ hospital sites), a prevalence of $27.4 \%$ was found [5]. In 2017, the second Global-PPS was set up.

The aim of this paper is to present the main results of the second ECDC-PPS and Global-PPS of antimicrobial use and HAIs in Belgian acute care hospitals in 2017.

\section{Methods}

\section{Study design and participation}

Two cross-sectional studies, the ECDC and Global-PPS, were simultaneously organized in Belgian acute care hospitals in 2017 by Sciensano and the Belgian Antibiotic Policy Coordination Committee (BAPCOC).
In order to collect data representative for the Belgian hospital population in the ECDC-PPS, a representative sample of hospitals was drawn using a systematic sampling design. For this, a list of all Belgian hospitals was obtained from the Federal Public Service health, Food chain safety and Environment [6]. All acute care hospitals were selected and ranked according to hospital type, total number of beds, region/province and ownership. The total number of hospitals $(N=102$ administrative hospital groups (AHG), i.e. hospital sites that are grouped) was divided by the number to be sampled $(N=34)$ to determine the sampling interval. One substitution per hospital (runner-up) was foreseen in case of refusal of the first selected hospital.

In collaboration with Sciensano, BAPCOC invited the infection control team members and representative(s) of the antibiotic policy group within each hospital to participate in one of the two surveys. The 34 randomly selected hospitals received from Sciensano a personalized invitation and were encouraged to participate in the ECDC-PPS. Because the more complex protocol compared to the Global-PPS and higher workload, financial incentives were given to hospitals conducting the ECDC-PPS (2 euros per included patient). All inclusions in the ECDC-PPS were performed between September and November 2017. The Global-PPS was performed between September and December 2017.

\section{Data collection}

The ECDC-PPS was organized in line with ECDC's patientbased protocol for PPS of HAI and antimicrobial use in European acute care hospitals [7]. The protocol of the Global-PPS is available on the Global-PPS website (www.global-pps.com).

\section{ECDC-PPS}

Data had to be collected on one single day for each ward in the participating hospitals. The entire data collection could not exceed 2-3 weeks in a single hospital. Accident and emergency wards were excluded. A hospital form collected data on hospital type, size, beds and patients as well as a limited set of structure and process indicators (including the full-time equivalent (FTE) antimicrobial stewardship consultants, interpreted as the time that a consultant/pharmacist is specifically employed and paid for antimicrobial stewardship tasks [7]). For all patients present at the ward before or at 8 a.m. and not discharged from the ward at the time of the survey, a patient form had to be completed. This form collected patient demographic data and risk factors, use of antimicrobial agents and presence of HAIs.

Antimicrobial agents for systemic use within the Anatomical Therapeutic Chemical (ATC) groups A07AA (intestinal anti-infectives), D01BA (antifungals for systemic use), J01 (antibacterials for systemic use), J02 (antimycotics for systemic use), J04 (antimycobacterials) 
and P01AB (nitroimidazole-derived antiprotozoals) were included [8]. Antiviral agents (J05) and antimicrobials for the treatment of mycobacteria were excluded.

If the antimicrobial (or administration route) changed during the infection episode, the reason for change had to be registered. Possible reasons were escalation (i.e. another antimicrobial was added, the route of administration was switched from oral to parental), de-escalation (i.e. the antimicrobial was switched to a more narrow-spectrum or to a first-line antimicrobial, or other antimicrobials for the same indication were stopped), switch from intravenous to oral administration route, change because of observed or expected adverse effect of the antimicrobial and changes for another or unknown reason [7].

Infections had to be included if they met the definition of an active HAI (associated to an acute care hospital stay). An infection was considered to be active when signs and symptoms of the infection were present on the survey date or when the patient was (still) receiving treatment for that infection on the survey date. The onset of symptoms had to be on day 3 or later (day of admission = day 1) of the current admission or earlier in case the patient presenting an infection was readmitted less than $48 \mathrm{~h}$ after a previous admission to an acute care hospital. Exceptions to these inclusion criteria were there for SSIs, Clostridioides difficile infections and infections with invasive devices [7]. Microbiological test results available on the day of the PPS were collected. For a selected group of bug-drug combinations, the antimicrobial susceptibility test results (susceptible, intermediate, resistant or unknown) also had to be reported [7].

Prior to the start of the surveillance period (September 2017), several training days were organized to outline the PPS objectives and methodology to the participating hospitals. Local surveyors had to enter all data into ECDC's HelicsWin.Net software. Thereafter, the local database had to be sent to Sciensano. All individual databases were validated, compiled and transferred to ECDC using their European Surveillance System (TESSy). The data of hospitals that participated in the ECDC-PPS were afterwards converted and imported in the Global-PPS tool (more details on this conversion in Additional file 1).

\section{Global-PPS}

Participating hospitals were asked to conduct the survey on one single-day and audit all in-patient wards. All patients present in the ward at $8 \mathrm{a} . \mathrm{m}$. had to be included. Data were collected using two forms, a ward form for the recording of denominators (number of beds and number of admitted patients at $8 \mathrm{a} . \mathrm{m}$. on the day of the PPS) and a patient form for recording detailed antimicrobial prescription (type, dose, administration route, indication, diagnosis) and resistance data for those patients who received at least one antimicrobial on the day of the PPS.
The following ATC groups were included as antimicrobial agents for systemic use: A07AA, D01BA, J01, J02, J04A, J05, $\mathrm{P} 01 \mathrm{AB}$ and $\mathrm{P} 01 \mathrm{~B}$ (antimalarials). Additional antimicrobial quality indicators included 1) the diagnosis being documented in the patient's notes at the start of treatment; 2) the antibiotic prescription being compliant with local guidelines and 3) if a stop or review date of the antimicrobial prescription was documented in the notes. Further, empiric or targeted treatment (based upon microbiology data from a relevant clinical specimen) was recorded. If the treatment choice was determined by available microbiology data, the participant had to indicate if it targeted a multidrug-resistant organism. Data collection forms and definitions on the different variables are available on the Global-PPS website (www.global-pps.com).

The data were entered by the participating hospitals in the freely available web-based application of the Global-PPS. This system allows anonymised data entry, validation and feedback reporting [4]. The complete database is safeguarded at the University of Antwerp. The validated database of June 2018 was used for the analyses in this manuscript.

\section{Data analysis}

Where possible, the data of both databases were combined and total results are shown for the Global- and ECDC-PPS (except for data that were collected only in one of the PPS). For the section on HAIs, results of each PPS were separately reported due to the differences in the methodology.

Hospital sites were classified by type (primary, secondary, tertiary or specialized) in accordance with the recommendations of ECDC and based on the list of hospitals of the Federal Public Service Health, Food chain safety and Environment [6, 7]. Total results for the Global- and ECDC-PPS were presented per ward specialty (in line with the classification of the Global-PPS); specific results for the ECDC-PPS were presented by patient specialty.

The crude prevalence of patients with at least one HAI or antimicrobial was calculated by dividing the number of patients presenting at least one HAI or antimicrobial by the total number of eligible patients. Patients presenting with multiple HAIs or prescribed multiple antimicrobials on the PPS day were thus counted only once. Prevalences were calculated along with their 95\%CI.

Statistical testing was performed, using SAS Enterprise Guide statistical software, version 7.1. Means and standard deviations (SD), ranges and frequencies (\%) were calculated where appropriate. The data of the ECDC and Global-PPS on antimicrobial consumption and quality indicators were pooled.

\section{Results}

Characteristics of the participating hospitals and included patients

Overall, 110 acute care hospital sites participated in the Global-PPS and ECDC-PPS survey in 2017. Of the random 
Table 1 Characteristics of the included acute care hospitals in the Global and ECDC-PPS 2017 (Belgium)

\begin{tabular}{|c|c|c|c|c|c|c|c|c|}
\hline \multirow[t]{2}{*}{ Number of included } & \multicolumn{2}{|c|}{ Global-PPS 2017} & \multicolumn{2}{|c|}{ ECDC-PPS 2017} & \multicolumn{2}{|l|}{ Total } & \multicolumn{2}{|c|}{ Degree of participation ${ }^{a}$} \\
\hline & sites & $\mathrm{AHG}^{\mathrm{b}}$ & sites & $A H G^{b}$ & sites & $\overline{A H G^{b}}$ & sites & $\mathrm{AHG}^{\mathrm{b}}$ \\
\hline Total & 64 & 51 & 47 & 33 & 110 & 83 & $110^{c} / 191=57.6 \%$ & $83 / 102=81.4 \%$ \\
\hline \multicolumn{9}{|l|}{ Per type } \\
\hline Primary hospitals & 48 & 40 & 33 & 23 & 81 & 63 & $81 / 144=56.3 \%$ & $63 / 77=81.8 \%$ \\
\hline Secondary hospitals & 12 & 7 & 12 & 8 & 23 & 14 & $23 / 27=85.2 \%$ & $14 / 17=82.4 \%$ \\
\hline Tertiary hospitals & 3 & 3 & 2 & 2 & 5 & 5 & $5 / 9=55.6 \%$ & $5 / 7=71.4 \%$ \\
\hline Specialized hospitals & 1 & 1 & 0 & 0 & 1 & 1 & $1 / 11=9.1 \%$ & $1 / 1=100.0 \%$ \\
\hline \multicolumn{9}{|l|}{ Per region } \\
\hline Brussels & 11 & 6 & 7 & 6 & 17 & 11 & $17 / 23=73.9 \%$ & $11 / 12=91.7 \%$ \\
\hline Flanders & 33 & 30 & 16 & 12 & 49 & 42 & $49 / 101=48.5 \%$ & $42 / 54=77.8 \%$ \\
\hline Wallonia & 20 & 15 & 24 & 15 & 44 & 30 & $44 / 67=65.7 \%$ & $30 / 36=83.3 \%$ \\
\hline
\end{tabular}

AHG Administrative hospital groups, PPS Point prevalence survey

a Based on the total number of hospital sites in Belgium in 2017 (6) (total AHG: N=102; total sites; $n=191$ )

${ }^{\mathrm{b}}$ At least one site of the AHG participated

'One hospital participated both in the Global and ECDC-PPS 2017 (other point in time, other patients)

selection ( $N=34$ AHG), 16 primarily selected hospitals and 6 runner-ups participated in the ECDC-PPS. In total, 28, 007 patients were included, of whom 16,207 patients in the Global-PPS and 11,800 patients in the ECDC-PPS. The characteristics of these hospital sites and their eligible patients are displayed in Tables 1 and 2, respectively.

\section{Antimicrobial consumption}

The overarching results of the Global-PPS and ECDCPPS will be jointly presented. Overall, the crude prevalence of patients with at least one antimicrobial was 27.1\% (95\%CI: 26.5-27.6\%). In total, 7577 patients used one or more antimicrobials on the survey day. These patients had a mean age of 64.8 (SD: \pm 21.8 ) years and $51.3 \%$ was male. Table 3 presents the crude prevalence by ward specialty, antimicrobial subclass and hospital type. The prevalence by hospital site ranged from 2.2 to 43.3\% (interquartile range: $23.0-31.4 \%$ ).

Moreover, 1378 patients (18.2\%) were treated with multiple antimicrobials on the day of the PPS. Out of all antimicrobials $(N=9232), 51.7 \%$ were prescribed for a communityacquired infection (CAI, $N=4775$ ), $25.3 \%$ for a healthcareassociated infection (HAI, $N=2333$ ), $2.7 \%$ for an infection related to a long-term care facility (LAI, $N=248$ ), $5.9 \%$ for medical $(N=545)$ and $11.2 \%$ for surgical prophylaxis $(N=$ 1038). Out of all antimicrobials, 8448 were antibacterials for systemic use $(91.5 \%)$ for which antibiotic subclasses by indication are provided in Fig. 1. More details can be found in the supplementary data (Additional file 2). For therapeutic indications (CAI, HAI, LAI) especially 'Penicillins in combination with a beta-lactamase inhibitor' (J01CR; 30.1-40.2\% of J01) and 'Fluoroquinolones' (J01MA, 14.6-16.2\% of J01) were used. For medical prophylaxis, 'Combinations of sulfonamides and trimethoprim' (J01EE, 27.5\% of J01) was the most used antibiotic subgroup. 'First-generation cephalosporines' (J01DB, $69.2 \%$ of J01, especially cefazolin) was most prescribed for surgical prophylaxis.

The five most frequent diagnoses for medical treatment with antimicrobials (CAI, HAI, LAI) were pneumonia $(N=1705,23.2 \%)$, (lower and upper) UTIs $(N=1121$, $15.2 \%)$, skin and soft tissue infections $(N=877,11.9 \%)$, intra-abdominal sepsis $(N=781,10.6 \%)$ and acute bronchitis $(N=522,7.1 \%)$. Overall, the following antimicrobials were most commonly used: amoxicillin/betalactamase inhibitor $(N=1935,21.0 \%)$, piperacillin/betalactamase inhibitor $(N=781,8.5 \%)$, cefazolin $(N=726$, $7.9 \%)$, ciprofloxacin $(N=664,7.2 \%)$ and ceftriaxone $(N=$ $350,3.8 \%)$. Of all antimicrobial agents, $64.6 \%$ was administered parenterally $(N=5966) 35.2 \%$ orally $(N=3248)$, $0.13 \%$ by inhalation $(N=12)$ and $0.01 \%$ rectally $(N=1$; missing: $N=5$ ). More details on the diagnosis sites by indication can be found in the supplementary data (Additional file 3).

\section{Antimicrobial quality indicators}

Antimicrobial quality indicators are presented by hospital type, ward specialty and indication in Table 4. In general, the reason for antimicrobial use was recorded for $81.9 \%$ of the prescriptions and a stop/review date was known for $40.8 \%$ of the prescriptions. For antibiotic prescriptions, a compliance with local antibiotic guidelines was reported in $76.6 \%$. For surgical prophylaxis, this compliance was $73.2 \%$. The duration of surgical prophylaxis was in $35.1 \%$ of the cases a single dose, in $39.7 \% 1$ day (multiple doses) and in $25.2 \%$ more than 1 day. For prolonged surgical prophylaxis ( $>1$ day), prophylaxis for plastic or orthopedic surgery (30.8\%) and prophylaxis for urological surgery $(27.7 \%)$ were most often registered as diagnosis (only registered in the Global-PPS). 
Table 2 Characteristics of the eligible patients in the Global and ECDC-PPS 2017 (Belgium, acute care hospitals)

\begin{tabular}{|c|c|c|c|c|c|c|}
\hline & \multicolumn{2}{|c|}{ Global-PPS 2017} & \multicolumn{2}{|c|}{ ECDC-PPS 2017} & \multicolumn{2}{|l|}{ Total } \\
\hline & $\mathrm{N}$ & $\%$ & $\mathrm{~N}$ & $\%$ & $\mathrm{~N}$ & $\%$ \\
\hline Total number of included patients & 16,207 & 100.0 & 11,800 & 100.0 & 28,007 & 100.0 \\
\hline Adults $^{\mathrm{a}}$ & 15,139 & 93.4 & 11,008 & 93.3 & 26,147 & 93.4 \\
\hline Children $^{a}$ & 722 & 4.5 & 606 & 5.1 & 1328 & 4.7 \\
\hline Neonates $^{a}$ & 346 & 2.1 & 186 & 1.6 & 532 & 1.9 \\
\hline Mean age $\pm S D^{b}$ & & & $60.2 \pm 25.3$ & & & \\
\hline $\begin{array}{l}\text { Number of males / females } \\
\text { Missing }\end{array}$ & & & $\begin{array}{l}5264 / 6512 \\
24\end{array}$ & $\begin{array}{l}44.7 / 55.2 \\
0.2\end{array}$ & & \\
\hline \multicolumn{7}{|l|}{ Distribution of the McCabe score (\%) } \\
\hline Non-fatal disease & & & 7295 & 61.8 & & \\
\hline Ultimately fatal disease & & & 1873 & 15.9 & & \\
\hline Rapidly fatal disease & & & 689 & 5.8 & & \\
\hline Missing & & & 1943 & 16.5 & & \\
\hline \multicolumn{7}{|l|}{ Ward specialty (\%) } \\
\hline Medicine & 11,067 & 68.3 & 8837 & 74.9 & 19,904 & 71.1 \\
\hline Surgery & 4293 & 26.5 & 2432 & 20.6 & 6725 & 24.0 \\
\hline $\mathrm{ICU}$ & 847 & 5.2 & 531 & 4.5 & 1378 & 4.9 \\
\hline \multicolumn{7}{|l|}{ Patient specialty $(\%)^{b}$} \\
\hline Medicine & & & 3600 & 30.5 & & \\
\hline Surgery & & & 2531 & 21.4 & & \\
\hline ICU & & & 583 & 4.9 & & \\
\hline Geriatrics & & & 1813 & 15.4 & & \\
\hline Obstetrics / Maternity & & & 583 & 4.9 & & \\
\hline Healthy neonates & & & 156 & 1.3 & & \\
\hline Neonatology & & & 121 & 1.0 & & \\
\hline Pediatrics & & & 464 & 3.9 & & \\
\hline Psychiatry & & & 823 & 7.0 & & \\
\hline Rehabilitation & & & 903 & 7.7 & & \\
\hline Long-term care & & & 33 & 0.3 & & \\
\hline Mix & & & 28 & 0.2 & & \\
\hline Other & & & 50 & 0.4 & & \\
\hline Missing & & & 112 & 1.0 & & \\
\hline
\end{tabular}

ECDC European Centre for Disease Prevention and Control, ICU Intensive care unit, $N$ number of patients, PPS point prevalence survey, SD Standard deviation ${ }^{a}$ The classification of adults, children and neonates is based on the ward type

${ }^{b}$ These data are only available in the ECDC-PPS whereby patient characteristics were collected for all eligible patients (as opposed to the Global-PPS where this data was only available for patients who received at least one antimicrobial)

If data were not collected in the PPS, fields were left blank.

In the Global-PPS, $37.9 \%$ of therapeutic antimicrobial prescribing (CAI, HAI, LAI) was based on a microbiological result (targeted treatment $(N=1566)$, whereby following resistant micro-organisms were most often reported: extended-spectrum beta-lactamase (ESBL) producing Enterobacteriaceae ( $N=76$ patients), third generation cephalosporin resistant Enterobacteriaceae (non-ESBL producing or ESBL status unknown) $(N=39$ patients) and methicillin-resistant Staphylococcus aureus (MRSA) ( $N=26$ patients).
In the ECDC-PPS, a change in the antimicrobial treatment was registered for $24.7 \%$ of the antimicrobials $(N=1012)$ : escalation in 425 cases (10.4\%), switch from intravenous to oral in 256 cases (6.2\%), de-escalation in 237 cases (5.8\%), change due to an adverse effect in 21 cases $(0.5 \%)$, and change for another of unknown reason in 73 cases $(1.8 \%)$.

The median number of FTEs for antimicrobial stewardship consultants, registered in hospitals participating in the ECDC-PPS $(N=29)$, was 0.29 per 250 beds (interquartile range $0.20-0.55)$. 
Table 3 Crude prevalence of antimicrobial use by ward specialty/subclass/hospital type; total results for Global and ECDC-PPS 2017 (Belgium, acute care hospitals)

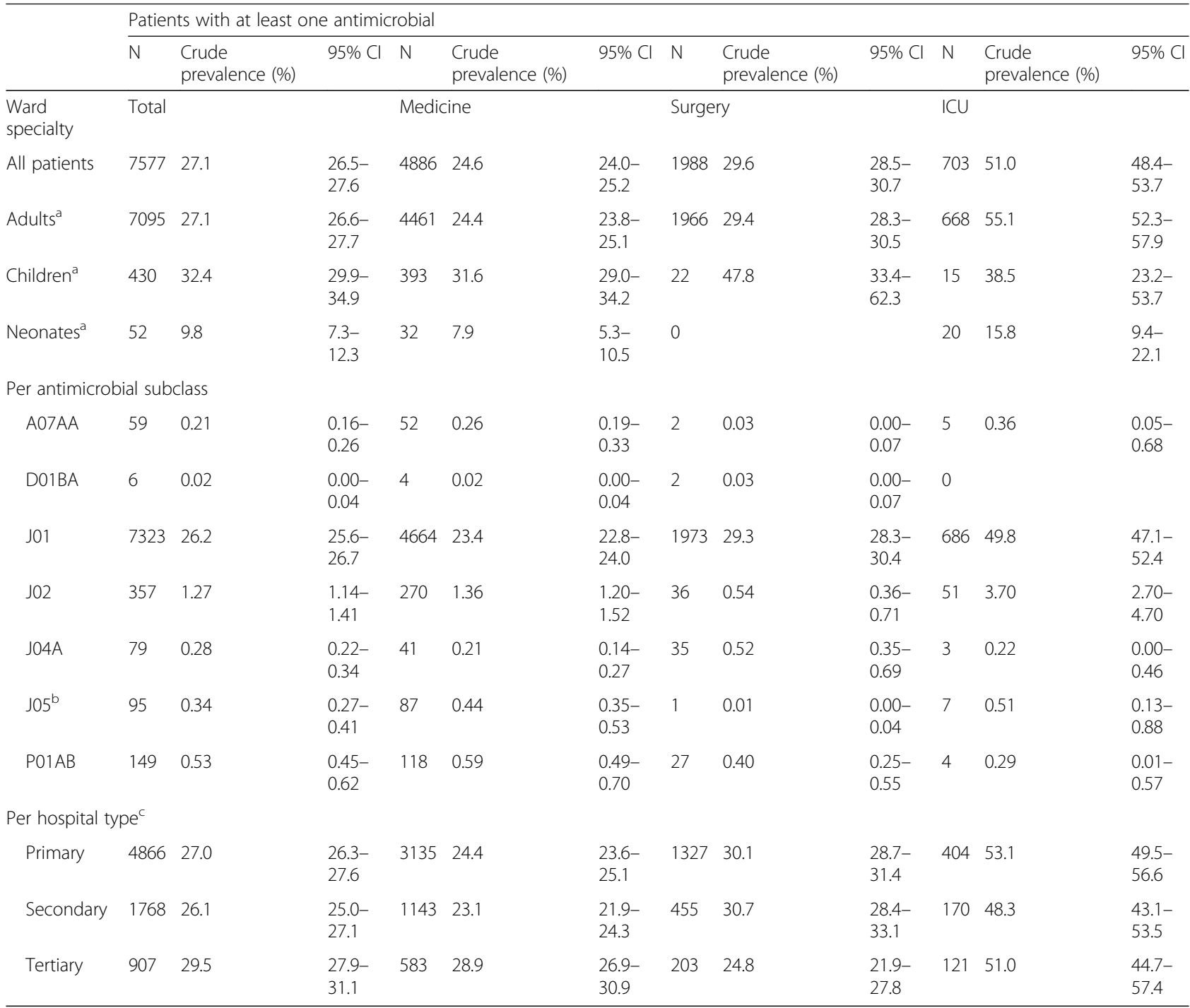

CI Confidence interval, ECDC European Centre for Disease Prevention and Control, ICU Intensive care units, $N$ Total number of patients with at least one antimicrobial, PPS Point prevalence survey

A07AA intestinal antiinfectives, D01BA antifungals for systemic use, $J 01$ antibacterials for systemic use, $J 02$ antimycotics for systemic use, $J 04 A$ drugs for treatment of tuberculosis, $J 05$ antivirals agents, $P 01 A B$ nitroimidazole-derived antiprotozoals ${ }^{a}$ The classification of adults, children and neonates is based on the ward type

bJ05 only included in the Global-PPS

${ }^{\mathrm{C}}$ Results of the specialized hospital $(N=1)$ not shown

\section{Healthcare-associated infections ECDC-PPS 2017}

In the subset of 47 hospital sites (11,800 included patients) that participated in the ECDC-PPS 2017, the crude prevalence of patients with at least one HAI was 7.3\% (95\%CI $6.8-7.7 \%$, range per hospital site: $0.0-$ $18.1 \%)$. The crude prevalences are shown by hospital type and patient specialty in Table 5. For 5.6\% (48/856) of the patients with at least one HAI, no antimicrobial medical treatment (prophylaxis excluded) was registered. Based on the indication of antimicrobial prescriptions, the prevalence of patients with a treatment for a HAI was $6.8 \%$ (95\% CI 6.3-7.2\%).

In total, 911 HAIs were registered of which $17.9 \%$ $(N=163)$ were already present on hospital admission. In most cases, the HAI was linked to the current hospital $(N=810,88.9 \%)$ and with the current ward $(N=617$, $67.7 \%)$. In $20.9 \%(N=190)$ of the cases, the HAI was linked to an invasive device. In Table 6 , the main groups of HAIs are displayed by patient specialty. The most common HAIs were pneumonia $(N=197,21.6 \%)$ and UTIs $(N=194,21.3 \%)$. 


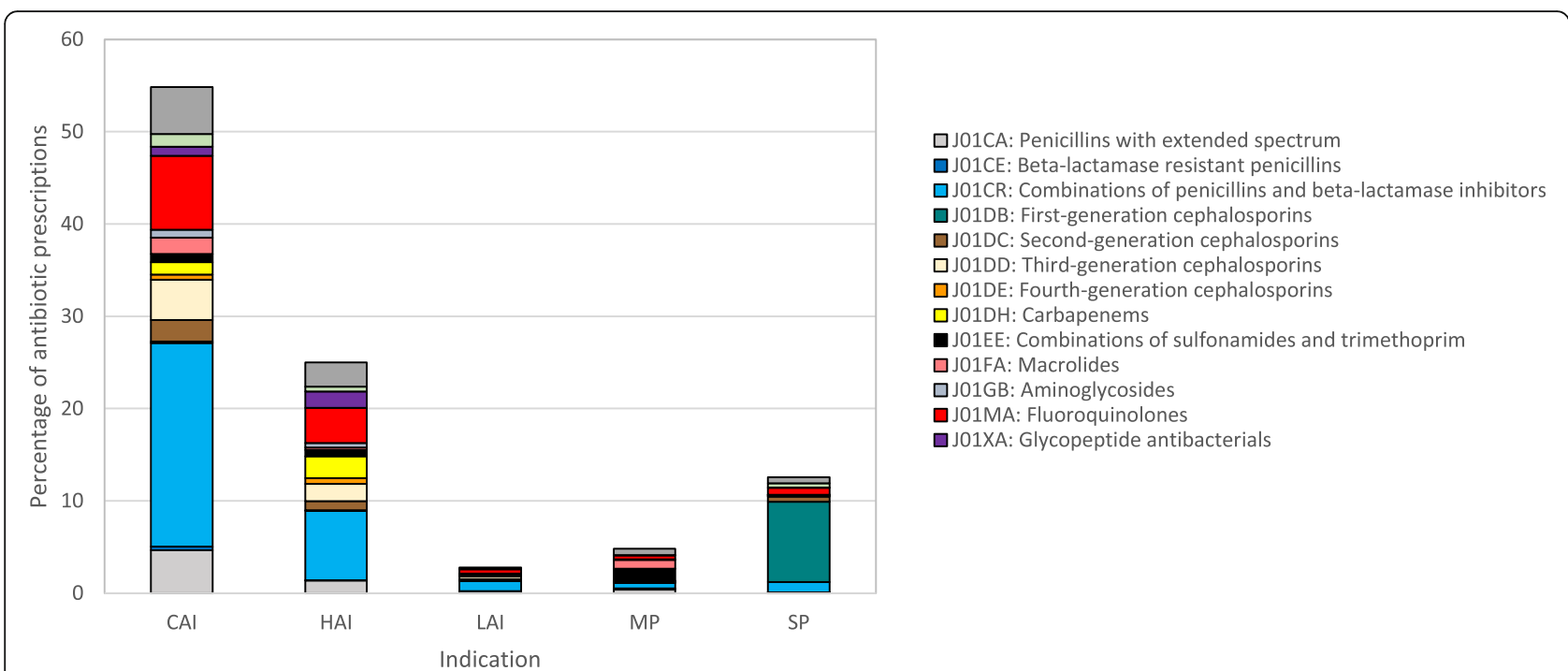

Fig. 1 Percentage of antibiotic prescriptions per antibiotic subclass and indication, total results for Global and ECDC-PPS 2017 (Belgium, acute care hospitals). CAI = community-acquired infection, $\mathrm{HAl}=$ acute-hospital-acquired infection, $\mathrm{LAl}=$ infection acquired in long-term care facility or chronic-care hospital, MP = medical prophylaxis, SP = surgical prophylaxis. * sum of the \% prescriptions CAI - HAl - LAI - MP $-\mathrm{SP}=100 \%$

Table 4 Overview of the antimicrobial quality indicators, total results for Global and ECDC-PPS 2017 (Belgium, acute care hospitals)

\begin{tabular}{|c|c|c|c|c|c|c|c|c|c|c|c|c|c|c|}
\hline & \multicolumn{2}{|c|}{$\begin{array}{l}\text { Targeted } \\
\text { treatment } \\
\end{array}$} & \multicolumn{2}{|c|}{$\begin{array}{l}\text { Targeted treatment } \\
\text { (resistant MO) }\end{array}$} & \multicolumn{2}{|c|}{$\begin{array}{l}\text { Reason } \\
\text { recorded }\end{array}$} & \multicolumn{2}{|c|}{$\begin{array}{l}\text { Stop or review } \\
\text { date recorded }\end{array}$} & \multicolumn{2}{|c|}{$\begin{array}{l}\text { Parenteral } \\
\text { administration }\end{array}$} & \multicolumn{2}{|c|}{$\begin{array}{l}\text { Guidelines } \\
\text { available }\end{array}$} & \multicolumn{2}{|c|}{$\begin{array}{l}\text { Compliant to local } \\
\text { guidelines }\end{array}$} \\
\hline & $\mathrm{N}$ & $\%$ & $\mathrm{~N}$ & $\%$ & $\mathrm{~N}$ & $\%$ & $\mathrm{~N}$ & $\%$ & $\mathrm{~N}$ & $\%$ & $\mathrm{~N}$ & $\%$ & $\mathrm{~N}$ & $\%$ \\
\hline All hospital sites & 1242 & 37.0 & 226 & 6.7 & 7558 & 81.9 & 2093 & 40.8 & 5072 & 69.3 & 3686 & 88.4 & 2825 & 76.6 \\
\hline \multicolumn{15}{|c|}{ Per type of hospital ${ }^{a}$} \\
\hline Primary & 804 & 33.9 & 156 & 6.6 & 4754 & 83.2 & 1362 & 39.8 & 3280 & 69.3 & 2508 & 86.1 & 1935 & 77.2 \\
\hline Secondary & 248 & 44.4 & 31 & 5.6 & 1860 & 84.8 & 480 & 53.0 & 1162 & 68.1 & 689 & 95.8 & 569 & 82.6 \\
\hline Tertiary & 185 & 45.7 & 37 & 9.1 & 889 & 70.1 & 237 & 32.0 & 606 & 71.4 & 455 & 89.9 & 289 & 63.5 \\
\hline \multicolumn{15}{|l|}{ Ward specialty } \\
\hline Medicine & 821 & 34.6 & 125 & 5.3 & 5007 & 84.5 & 1282 & 39.3 & 2911 & 62.4 & 2353 & 88.9 & 1820 & 77.3 \\
\hline Surgery & 279 & 43.3 & 60 & 9.3 & 1726 & 73.3 & 600 & 45.8 & 1501 & 76.1 & 969 & 87.2 & 703 & 72.5 \\
\hline ICU & 142 & 42.0 & 41 & 12.1 & 825 & 86.4 & 211 & 38.2 & 660 & 96.2 & 364 & 88.1 & 302 & 83.0 \\
\hline \multicolumn{15}{|l|}{ Indication } \\
\hline CAI & 684 & 30.7 & 89 & 4.0 & 4308 & 90.2 & 1001 & 37.5 & 2647 & 68.0 & 2021 & 90.3 & 1626 & 80.5 \\
\hline $\mathrm{HAl}$ & 523 & 51.1 & 129 & 12.6 & 2011 & 86.2 & 551 & 41.1 & 1250 & 71.4 & 911 & 86.7 & 713 & 78.3 \\
\hline LAI & 35 & 34.7 & 8 & 7.9 & 231 & 93.1 & 64 & 53.8 & 137 & 68.2 & 92 & 89.3 & 76 & 82.6 \\
\hline MP & NA & NA & NA & NA & 305 & 56.0 & 56 & 19.4 & 93 & 28.4 & 131 & 74.4 & 88 & 67.2 \\
\hline SP & NA & NA & NA & NA & 640 & 61.7 & 377 & 82.3 & 859 & 89.5 & 414 & 91.6 & 303 & 73.2 \\
\hline
\end{tabular}

ECDC European Centre for Disease Prevention and Control, CAI Community-acquired infections, HAI Healthcare-associated infections, ICU Intensive care unit, LAI Infection present on admission from long-term care facility or Nursing Home, MO Micro-organisms, MP Medical prophylaxis, $N$ Number of prescriptions or patients, NA Not applicable, PPS Point prevalence survey, SP Surgical prophylaxis

${ }^{a}$ Results of the specialized hospital $(N=1)$ not shown

Targeted treatment: only patients included in the Global-PPS and with at least one antibiotic medical (CAl, HAl, LAI) treatment $(N=3354)$

Reason recorded: patients of the Global- and ECDC-PPS; all antimicrobial prescriptions included

Stop or review date recorded: only patients included in the Global-PPS; all antimicrobial prescriptions included

Parenteral administration: patients of the Global- and ECDC-PPS; patients who received at least one parenteral antibiotic for systemic use over all patients who received at least one antibiotic for systemic use

Guidelines available: only patients included in the Global-PPS; antibiotic prescriptions for which guidelines were available, count at patient level and diagnosis (for combination therapy: no guidelines available for $\geq 1$ antibiotic = no guideline for combination)

Compliant to local guidelines: only patients included in the Global-PPS; antibiotic prescriptions for which guidelines were available used as denominator, count at patient level and diagnosis (for combination therapy no compliance for $\geq 1$ antibiotic $=$ combination not compliant) 
Table 5 Crude prevalence of HAl and antimicrobial use, ECDC-PPS 2017 (Belgium, acute care hospitals)

\begin{tabular}{|c|c|c|c|c|}
\hline & \multirow{2}{*}{$\begin{array}{l}\text { Total } \\
\text { number } \\
\text { of } \\
\text { patients }\end{array}$} & \multicolumn{3}{|c|}{ Patients with at least one $\mathrm{HAl}$} \\
\hline & & $\mathrm{N}$ & Crude prevalence (\%) & $95 \% \mathrm{Cl}$ \\
\hline Total prevalence & 11,800 & 856 & 7.3 & $6.8-7.7$ \\
\hline Total prevalence with exclusion of psychiatry, rehabilitation and long-term care & 10,041 & 776 & 7.7 & $7.2-8.3$ \\
\hline \multicolumn{5}{|l|}{ Prevalence by hospital type } \\
\hline Primary & 7214 & 489 & 6.8 & $6.2-7.4$ \\
\hline Secondary & 3337 & 253 & 7.6 & $6.7-8.5$ \\
\hline Tertiary & 1249 & 114 & 9.1 & $7.5-10.7$ \\
\hline \multicolumn{5}{|l|}{ Prevalence by patient specialty } \\
\hline Medicine & 3600 & 265 & 7.4 & $6.5-8.2$ \\
\hline Surgery & 2531 & 204 & 8.1 & $7.0-9.1$ \\
\hline Intensive care & 583 & 122 & 20.9 & $17.6-24.2$ \\
\hline Geriatrics & 1813 & 158 & 8.7 & $7.4-10.0$ \\
\hline Obstetrics/ Maternity & 583 & 9 & 1.5 & $0.5-2.5$ \\
\hline Healthy neonates & 156 & 0 & 0.0 & \\
\hline Neonatology & 121 & 4 & 3.3 & $0.1-6.6$ \\
\hline Pediatrics & 464 & 12 & 2.6 & $1.1-4.0$ \\
\hline Psychiatry & 823 & 9 & 1.1 & $0.4-1.8$ \\
\hline Rehabilitation & 903 & 67 & 7.4 & $5.7-9.1$ \\
\hline Long-term care & 33 & 4 & 12.1 & $1.0-23.3$ \\
\hline Mix & 28 & 0 & 0.0 & \\
\hline Other & 50 & 2 & 4.0 & $0.0-9.4$ \\
\hline
\end{tabular}

CI Confidence interval, ECDC European Centre for Disease Prevention and Control, HAl Healthcare-associated infection, $N$ Number of patients with at least one HAl/antimicrobial, PPS Point prevalence survey

A positive microbiological result was reported for $62.0 \%$ of the HAIs $(N=565 / 911)$. In total, $721 \mathrm{mi}-$ croorganisms were documented. The most commonly isolated micro-organisms were Escherichia coli $(N=162,17.8 \%)$, Staphylococcus aureus $(\mathrm{N}=81$, 8.9\%) and Pseudomonas aeruginosa $(\mathrm{N}=47,5.2 \%)$. In the susceptibility tests, a resistant result was reported for 97 tests (susceptible: $N=793$, intermediate: $\mathrm{N}=3$, not available or unknown: $N=454$ ). More details on the intermediate and resistant microorganisms are presented in Additional file 4.

\section{Global-PPS 2017}

In the Global-PPS 2017 (64 hospital sites, 16,207 included patients), the prevalence of patients with at least one HAI (based on the indication of antimicrobial prescriptions) was $6.8 \%$ (95\%CI $6.4-7.2 \%$, hospital site range: $2.0-12.1 \%$ ). Similar as in the ECDC-PPS, the HAI prevalence was highest in tertiary hospitals $(10.6 \%$ [95\%CI $9.2-12.0 \%])$ and in ICU $(22.0 \%$ [95\%CI $19.2-24.8 \%])$.

\section{Discussion}

Main results and comparison with previous PPS in Belgian acute care hospitals

Prevalence of antimicrobial consumption

A crude prevalence of patients with at least one antimicrobial of $27.1 \%$ was found. This prevalence is slightly lower than the Belgian results in the previous ECDC-PPS (28.9\%) and Global-PPS (27.4\%) conducted in 2011 and 2015, respectively. The proportion of medical (5.9\%) and surgical prophylaxis (11.2\%) decreased in comparison with 2011 (9.0\% and $11.8 \%$ respectively). The top 3 of most used antimicrobial agents (amoxicillin/beta-lactamase inhibitor, piperacillin/beta-lactamase inhibitor, cefazolin) and the percentage of antimicrobials administered parenterally (64.6\%) remained the same as in 2011 and 2015 [3, 5]. In the participating European hospitals in the ECDC-PPS 2016-2017, a higher percentage of antimicrobials were administered parenterally (72.8\%) [9]. These results also correspond with the results of the national surveillance of antimicrobial consumption in Belgian hospitals (BeH-SAC) $[10,11]$. 
Table 6 Distribution of main groups of HAls by patient specialty, ECDC-PPS 2017 (Belgium, acute care hospitals)

\begin{tabular}{|c|c|c|c|c|c|c|c|c|c|c|c|c|c|c|c|c|}
\hline \multirow{2}{*}{$\begin{array}{l}\text { Patient } \\
\text { Specialty }\end{array}$} & \multicolumn{15}{|c|}{ Number (\%) of infections by main HAl group } & \multirow[t]{2}{*}{ Total $(\%$} \\
\hline & BJ & BSI & CNS & CRI & CVS & EENT & $\mathrm{Gl}$ & LRTI & NEO & PN & REPR & SSI & SST & SYS & UTI & \\
\hline Medicine & $2(0.7)$ & $40(14.2)$ & & $7(2.5)$ & $3(1.1)$ & $10(3.6)$ & $32(11.4)$ & $10(3.6)$ & & $69(24.6)$ & $1(0.4)$ & $16(5.7)$ & $14(5.0)$ & $20(7.1)$ & $57(20.3)$ & $281(30.8)$ \\
\hline Surgery & $3(1.4)$ & $21(9.6)$ & $3(1.4)$ & $3(1.4)$ & $2(0.9)$ & $1(0.5)$ & $15(6.9)$ & $2(0.9)$ & & $20(9.1)$ & $1(0.5)$ & $95(43.4)$ & $8(3.7)$ & $6(2.7)$ & $39(17.8)$ & $219(24.0)$ \\
\hline Intensive care & & $24(17.8)$ & $1(0.7)$ & $2(1.5)$ & $3(2.2)$ & & $9(6.7)$ & $14(10.4)$ & & $49(36.3)$ & & $15(11.1)$ & $1(0.7)$ & $5(3.7)$ & $12(8.9)$ & $135(14.8)$ \\
\hline Geriatrics & & $15(9.0 \%)$ & & $2(1.2)$ & & $3(1.8)$ & $22(13.3)$ & $10(6.0)$ & & $43(25.9)$ & & $5(3.0)$ & $7(4.2)$ & $5(3.0)$ & $54(32.5)$ & $166(18.2)$ \\
\hline $\begin{array}{l}\text { Obstetrics/ } \\
\text { Maternity }\end{array}$ & & & & & & & & & & $1(10.0)$ & & $5(50.0)$ & $1(10.0)$ & & $3(30.0)$ & $10(1.1)$ \\
\hline $\begin{array}{l}\text { Healthy } \\
\text { neonates }\end{array}$ & & & & & & & & & & & & & & & & 0 \\
\hline Neonatology & & $1(25.0)$ & & & & & & $1(25.0)$ & & $1(25.0)$ & & & & $1(25.0)$ & & $4(0.4)$ \\
\hline Pediatrics & & $1(8.3)$ & & & & $2(16.7)$ & $3(25.0)$ & $1(8.3)$ & & $1(8.3)$ & & $2(16.7)$ & & $1(8.3)$ & $1(8.3)$ & $12(1.3)$ \\
\hline Psychiatry & & & & & & & & $2(22.2)$ & & $1(11.1)$ & & & $2(22.2)$ & & $4(44.4)$ & $9(1.0)$ \\
\hline Rehabilitation & $1(1.5)$ & $2(2.9)$ & & & $1(1.5)$ & $2(2.9)$ & $5(7.4)$ & $5(7.4)$ & & $11(16.2)$ & & $14(20.6)$ & $1(1.5)$ & $2(2.9)$ & $24(35.3)$ & $68(7.5)$ \\
\hline $\begin{array}{l}\text { Long-term } \\
\text { care }\end{array}$ & & $1(20.0)$ & & & & & & & & $1(20.0)$ & & $2(40.0)$ & $1(20.0)$ & & & $5(0.5)$ \\
\hline Mix & & & & & & & & & & & & & & & & 0 \\
\hline Other & & & & & & $1(50.0)$ & $1(50.0)$ & & & & & & & & & $2(0.2)$ \\
\hline Total & $6(0.7)$ & $105(11.5)$ & $4(0.4)$ & $14(1.5)$ & $9(1.0)$ & $19(2.1)$ & $87(9.6)$ & $45(4.9)$ & 0 & 197 (21.6) & $2(0.2)$ & $154(16.9)$ & $35(3.8)$ & $40(4.4)$ & $194(21.3)$ & 911 \\
\hline
\end{tabular}

BJ Bone and joint infection, BSI Bloodstream infection, CNS Central nervous system infection, CRI Catheter-related infection, CVS Cardiovascular infection, ECDC European Centre for Disease Prevention and Control, EENT Eye, ear, nose or mouth infection, GI Gastro-intestinal infection, HAI Healthcare-associated infection, $L R T I$ Lower respiratory tract infection, $N$ Number of infections, NEO Specific neonatal cases, PN Pneumonia, PPS Point prevalence survey, REPR Reproductive tract infection, SSI Surgical site infection, SST Skin and soft tissue infection, SYS Systemic infection, UTI Urinary tract infection

\section{Antimicrobial quality indicators}

BAPCOC published an action plan 2014-2019 with specific targets for the hospital setting that should be accomplished by 2019 [12]. The first target is that $90 \%$ of antibiotic prescriptions should be in line with the local guidelines. In the current study, a compliance of $76.6 \%$ was reported. This compliance was lowest in tertiary hospitals $(63.5 \%$, but the highest proportion of targeted treatment: $45.7 \%)$ and for medical prophylaxis (67.2\%). Only in $88.4 \%$ of the prescriptions it was indicated that a local guideline was available. In the Global-PPS 2015, guideline availability (90.8\%) and compliance (79.5\%) was slightly higher [5].

Specifically for surgical prophylaxis, 90\% compliance with local guidelines for both the choice and duration of treatment is targeted by BAPCOC [12]. In 2017, the compliance concerning the choice of drug was $73.2 \%$. First-generation cephalosporins (especially cefazolin) were prescribed in $69.2 \%$, followed by penicillins in combination with a beta-lactamase inhibitor (8.8\%) and fluoroquinolones (5.9\%). The duration of surgical prophylaxis was prolonged (> 1 day) in $25.2 \%$. In 2015 , the mean proportion of prolonged surgical prophylaxis cases was $28.2 \%$ and cefazolin was prescribed in $62.6 \%$ [5]. Penicillins with a beta-lactamase inhibitor and fluoroquinolones are still prescribed in a considerable amount of cases. BAPCOC performed audits on surgical prophylaxis in hospitals in 2013 and 2017; choice and duration compliance are expected to further improve. More detailed analyses are needed to allow target setting for specific indications.
The action plan of BAPCOC stated that the indication of the prescription should be available in the medical file in $90 \%$ [12]. This study shows that this target is not yet achieved (reason for antimicrobial use was recorded in $81.9 \%)$. Finally, post-prescription review is also an important antimicrobial quality indicator to prevent unnecessary prolonged antimicrobial use [13]. The GlobalPPS showed the availability of a stop/review date in only $40.8 \%$ of the prescriptions.

\section{Prevalence of healthcare-associated infections}

Based on the ECDC-PPS 2017 data, a crude prevalence of patients with at least one HAI of $7.3 \%$ was detected in Belgian acute care hospitals. The highest prevalences were found in tertiary hospitals (9.1\%) and on ICU (20.9\%). In comparison with the ECDC-PPS in 2011, this prevalence remained stable (7.1\%) [3]. The most frequently reported HAIs were pneumonia, UTIs and SSIs, which is in line with the previous PPS [3].

\section{Comparison with other European countries}

The overall prevalence of antimicrobial consumption in Belgian acute care hospitals (27.1\%) is lower than reported for the participating European hospitals in the ECDC-PPS 2016-2017 (weighted prevalence: 30.3\% [95\%CI 29.0-31.6\%]). In addition, the amount of prolonged surgical prophylaxis ( $>1$ day) in Belgian acute care hospitals $(25.2 \%)$ was clearly lower than in the participating European hospitals (54.2\%, country range 19.8-95.0\%) [9]. Belgian acute care hospitals reported a 
higher number of FTE for antimicrobial stewardship per 250 beds (Belgian median 0.29 versus the European median 0.08 , country range $0-0.60$ ) [9]. In contrary, the prevalence of patients with at least one HAI in Belgian acute care hospitals (7.3\%) remains clearly higher than in other European hospitals. A weighted prevalence of HAI of 5.5\% (95\%CI 4.5-6.6\%) was reported in the participating European hospitals in the ECDC-PPS 20162017 [14]. In Scottish hospitals which performed the ECDC-PPS in 2016, a prevalence of antimicrobial use of $35.7 \%$ (95\% CI $34.2-37.2 \%)$ and HAI prevalence of $4.6 \%$ $(95 \% \mathrm{CI} 4.1-5.1 \%)$ were detected in acute adult patients [15].

\section{Strengths and limitations}

In 2017, two PPS were performed in Belgian acute care hospitals in line with the international standardized methodology of ECDC-PPS and Global-PPS. The added value of combining the data of the two surveys is that it resulted in a large database with a participation rate of more than $80 \%$ of all Belgian acute care hospitals. Moreover, each PPS system has its own added value (e.g. ECDC-PPS: more detailed data on HAIs, Global-PPS: other quality indicators), both at local and national level, resulting in a more complete picture on the current practice in Belgian acute care hospitals and allowing international comparisons. To our knowledge, it is the first time that data of both PPS using a similar methodology are combined. All participating hospitals already received an individual feedback of their prevalence data, benchmarked with national results. Participation, if organised countrywide, is also recorded as a mandatory quality indicator to improve infection control in Belgian hospitals [16].

Some limitations should be acknowledged. As this was a cross-sectional survey, only prevalences can be reported and patients were not followed-up in time. Prevalence study only show a snapshot of the situation, therefore they are not ideal for measuring for example AMR. However, in addition to the PPS, different (mandatory and voluntary) surveillances on AMR and HAIs are conducted in Belgian hospitals (e.g. surveillances on blood-stream infections, Clostridioides difficile, SSI, MRSA, vancomycin resistant enterococci (VRE), multi-resistant Gram negative bacteria) [10]. Secondly, the results were not corrected for patient case mix or institutional factors. Therefore, comparison with the results of different PPS should be interpreted carefully as different hospitals participated each time. Detected differences between several PPS might be explained by differences in case mix. In addition, the definition of the type of hospitals might have been different in past PPS. Finally, although we managed to merge the data of both PPS in the best way (see Additional file 1), some differences in the protocols of the ECDC-PPS and Global-PPS could have had a small influence on the results (e.g. antivirals (J05, 95 prescriptions: 1.03\%) and antimalarials (P01B, 3 prescriptions: $0.03 \%$ ) were only included in the Global-PPS). As only one hospital participated in both surveys, a direct comparison of the results obtained from both study methods was not assessed here.

\section{Future perspectives}

It is recommended that PPS are repeatedly performed to follow-up the evolution over time and monitor the impact of antimicrobial stewardship and infection prevention programmes [1]. The Global-PPS tool is continuously available and hospitals themselves can decide how often they conduct a PPS. ECDC aims to organize a PPS every 5 years (third ECDC-PPS probably planned in 2022). On a national level, a fixed timeinterval (e.g. every 2 years) should be defined to be able to provide data for benchmarking and to evaluate targets for example as set up in the 2014-2019 action plan of BAPCOC. Since 2018, Belgian hospitals are partly financed based on the level of quality in their hospital (Pay for Performance project), and participation in a PPS is one of the conditions for the financing [17]. There is still a large range in the prevalence of antimicrobial consumption (2.2 to $67.3 \%)$ and of HAIs (0.0 to $18.1 \%$ ) between hospital sites. Hospitals with outlying results should further be targeted to help the local antibiotic policy teams develop specific antimicrobial stewardship and infection prevention programmes to improve.

In general, concerning the antimicrobial consumption, the high prescribing of 'Fluoroquinolones' (J01MA) is a concern and should be a target for intervention. In addition, the results of the discussed antimicrobial quality indicators should be further improved to reach the BAPCOC targets (90\% for all indicators). Moreover, the number of patients who develop a HAI in Belgian acute care hospitals remains high. The reasons for these high rates of HAIs should be further investigated.

\section{Conclusions}

In comparison with previous PPS, the prevalence of antimicrobial use and HAI and most results for the antimicrobial quality indicators remained status quo. Belgian hospitals should be further stimulated to regularly participate in a PPS and to set local targets for improving antibiotic prescribing and reducing HAI.

\section{Supplementary information}

Supplementary information accompanies this paper at https://doi.org/10. 1186/s13756-019-0663-7.

Additional file 1: Table S1. Overview how the ECDC-PPS data were converted in the Global-PPS database (Belgium, acute care hospitals, 2017). 
Additional file 2: Table S2. Overview of antibiotic (J01) prescriptions by antibiotic subclass (ATC level 4) and by indication, total results for Global and ECDC-PPS 2017 (Belgium, acute care hospitals).

Additional file 3: Table S3. Description of diagnosis sites of the antimicrobial prescriptions per indication, total results for Global and ECDC-PPS 2017 (Belgium, acute care hospitals).

Additional file 4: Table S4. Overview of the number of isolates (selected bug-drug combinations) with known antimicrobial susceptibility testing results (AST; first-level antimicrobial resistance (AMR) markers combined) for healthcare-associated infections (HAls) and resistant results to the antimicrobials included in the protocol, ECDC-PPS 2017 (Belgium, acute care hospitals).

\section{Abbreviations}

AHG: Administrative hospital groups; AMR: Antimicrobial resistance; ATC: Anatomical Therapeutic Chemical; BAPCOC: Belgian Antibiotic Policy Coordination Committee; BJ: Bone and joint infection; BSI: Bloodstream infection; CAl: Community-acquired infections; Cl: Confidence interval; CNS: Central nervous system infection; CRI: Catheter-related infection; CVS: Cardiovascular infection; ECDC: European Centre for Disease Prevention and Control; EENT: Eye, ear, nose or mouth infection; ESBL: Extendedspectrum beta-lactamase; EU: European Union; FTE: Full-time equivalent; Gl: Gastro-intestinal infection; HAl: Healthcare-associated infection; ICU: Intensive care unit; LAI: Infection present on admission from long-term care facility or Nursing Home; LRTI: Lower respiratory tract infection; MO: Micro-organisms; MP: Medical prophylaxis; MRSA: Methicillin-resistant Staphylococcus aureus; N: Number; NA: Not applicable; NEO: Specific neonatal cases; PN: Pneumonia; PPS: Point prevalence survey; REPR: Reproductive tract infection;" RTI: Respiratory tract infection; SD: Standard deviation; SP: Surgical prophylaxis; SSI: Surgical site infection; SST: Skin and soft tissue infection; SYS: Systemic infection; UTI: Urinary tract infection; VRE: Vancomycin resistant enterococci

\section{Acknowledgements}

The authors would like to acknowledge the contribution of all involved staff members in the participating hospitals.

Members of the Belgian Point Prevalence Survey Study Group:

Marie, Andre (CHA Vivalia, Libramont, Belgium)

Samy, Aouachria (CHC-Centre Hospitalier Saint Vincent et Sainte Elisabeth Rocourt, Belgium)

Mickael, Aoun (Institut Jules Bordet, Brussels, Belgium)

Kristof, Bafort (Mariaziekenhuis, Pelt, Belgium)

Sofie, Bartholomeus (Sint-Dimpna ziekenhuis, Geel, Belgium)

Sophie, Blumental (Hôpital Universitaire des Enfants Reine Fabiola, Brussels, Belgium)

Anaïs, Bothy (Centre de Santé des Fagnes, Chimay, Belgium)

Christiane, Brands (Ziekenhuis Netwerk Antwerpen (ZNA), Antwerp, Belgium)

Laetitia, Brassinne (Cliniques de l'Europe, Brussels, Belgium)

Caroline, Briquet (Cliniques Universitaires Saint-Luc, Brussels, Belgium)

Reinoud, Cartuyvels (Jessa ziekenhuis, Hasselt, Belgium)

Clara, Ceyssens (AZ Sint-Jozef, Malle, Belgium)

Sarah, Cooreman (AZ Monica, Antwerp, Belgium)

Pierre-Yves, Decleire (Cliniques du Sud Luxembourg - Vivalia, Arlon, Belgium)

Philippe, Declercq (St. Jozefskliniek, Izegem, Belgium)

Liesbeth, De Cooman (OLV Lourdes, Waregem, Belgium)

Inge, De Cuyper (AZ St. Rembert, Torhout, Belgium)

Dirk, Degraeve (St. Franciscus ziekenhuis, Heusden-Zolder, Belgium)
(Continued)

Bénédicte, Delaere (CHU UCL Namur, Yvoir, Belgium)

Mélanie, Delvallée (Chwapi, Tournai, Belgium)

Alexander, De Smet (Heilig Hart Ziekenhuis Lier, Lier, Belgium)

Marjoleine, Desmedt (AZ Jan Portaels, Vilvoorde, Belgium)

Victoria, Diaz (CHC Liège, Liège, Belgium)

\|lisei, Dragos (ASBL Jolimont - CH Nivelles, Nivelles, Belgium)

Thierry, Dugernier (Clinique Saint Pierre, Ottignies, Belgium)

Elodie, Elsen (CHU UCL Namur site de Sainte-Elisabeth, Namur, Belgium)

Lorenzo, Filippin (Epicura, Hornu, Belgium)

Eric, Firre (CHR de Liège, Liège, Belgium)

Johan, Frans (Imelda hospital, Bonheiden, Belgium)

Patrick, Gabriëls (Sint-Trudo hospital, Sint-Truiden, Belgium)

Philippe, Gadisseux (Centre Hospitalier Mouscron, Mouscron, Belgium)

Michele, Gerard (CHU Saint-Pierre, Brussels, Belgium)

Bart, Glibert (AZ Glorieux, Ronse, Belgium)

Truus, Goegebuer (AZ Sint-Maarten, Mechelen, Belgium)

Viviane, Gonissen (Centre Hospitalier Bois de l'Abbaye, Seraign, Belgium)

Antoine, Harrouk (Clinique de l'Europe, Brussels, Belgium)

Xavier, Holemans (Grand Hôpital de Charleroi, Charleroi, Belgium)

Aline, Honore (Centre Hospitalier Régionale de Huy, Huy, Belgium)

Louis, Ide (AZ Jan Palfijn, Ghent, Belgium)

Hilde, Jansens (University Hospital Antwerp, Edegem, Belgium)

François, Kidd (Groupe Jolimont, La Louvière, Belgium)

Annelies, Koch (AZ Sint-Blasius, Dendermonde, Belgium)

Deborah, Konopnicki (Saint-Pierre University Hospital, Université Libre de Bruxelles, Brussels, Belgium)

Philippe, Lefèvre (IFAC Vivalia, Marche en Famenne, Belgium)

Marc, Lespagnard (Klinik St. Josef, St. Vith, Belgium)

Sophie, Lorent (Erasme University Hospital, Brussels, Belgium)

Evelyne, Maillart (CHU Brugmann, Brussels, Belgium)

Martine, Mallet (AZ Oudenaarde, Oudenaarde, Belgium)

Samuel, Markowicz (CHU Charleroi (ISPPC), Charleroi, Belgium)

Delphine, Mathieu (CHU Tivoli, La Louvière, Belgium)

Philippe, Michel (CHR Verviers, Verviers, Belgium)

Séverine, Noirhomme (CHR Namur, Namur, Belgium)

Pauline, Papin (Clinique Saint-Jean, Brussels, Belgium)

Anne, Piette (AZ Alma, Eeklo, Belgium)

Leen, Pollet (AZ West, Veurne, Belgium)

Camelia, Rossi (CHU Ambroise Paré, Mons, Belgium)

Patricia, Schatt (Clinique Notre Dame de Grâce, Gosselies, Belgium)

Erica, Sermijn (ASZ Aalst, Aalst, Belgium)

Astrid, Sterckx (CHU Brugmann, Brussels, Belgium)

Sophia, Steyaert (AZ Maria Middelares, Ghent, Belgium)

Walter, Swinnen (AZ Sint-Blasius, Dendermonde, Belgium)

Liliana, Teixeira Lopes (CHU Brugmann, Brussels, Belgium) 
(Continued)

Inge, Thoelen (AZ Vesalius Hospital, Tongeren, Belgium)

Myriam, Turkova (Centre Hospitalier Bois de l'Abbaye, Seraign, Belgium)

Anne-Marie, Van den Abeele (Algemeen Ziekenhuis Sint-Lucas, Ghent, Belgium)

Valérie, van den Berg (Clinique André Renard, Herstal, Belgium)

Danielle, Van der beek (AZ Herentals, Herentals, Belgium)

Vanessa, Vanderper (AZ Nikolaas, Sint-Niklaas, Belgium)

Marc, Vandevelde (Ziekenhuis Maas en Kempen, Maaseik, Belgium)

Wouter, Vandewal (AZ Sint Lucas Brugge, Brugge, Belgium)

Bruno, Van Herendael (GZA hospitals, Antwerp, Belgium)

Geert, Vanheule (Sint Jozefkliniek Bornem \& Willebroek, Bornem, Belgium)

Frederik, Van Hoecke (Sint Andries Hospital, Tielt, Belgium)

Dana, Van Kerkhoven (AZ Turnhout, Turnhout, Belgium)

Ann, Van Liedekerke (AZ Sint-Elisabeth, Zottegem, Belgium)

Lorenz, Vanneste (AZ Groeninge, Kortrijk, Belgium)

Marc, Vekemans (Hôpitaux IRIS Sud, Brussels, Belgium)

Christelle, Vercheval (CHU Liège, Liège, Belgium)

Katia, Verhamme (OLV Hospital, Aalst, Belgium)

Thessa, Verniest (Heilig Hart ziekenhuis, Leuven, Belgium)

Veerle, Westerlinck (AZ Sint-Maarten, Mechelen, Belgium)

Ingrid, Wybo (UZ Brussel, Brussels, Belgium)

\section{Authors' contributions}

EV gathered the data for the ECDC PPS, carried out the statistical analyses and drafted the manuscript. $\mathrm{KL}$ conceptualized and designed the study, assisted in the data collection for the ECDC PPS, and reviewed and revised the manuscript. HG and KM reviewed and revised the manuscript. ND assisted in the data collection for the Global-PPS. BC assisted in the conceptualization of the study, supervised the data management, and reviewed and revised the manuscript. AV conceptualized and designed the study, gathered the data for the Global-PPS, assisted with the statistical analyses, and reviewed and revised the manuscript. The Belgian Point Prevalence Survey Study Group consists of the responsible persons for the data collection and validation of each participating hospital. All authors read and approved the final manuscript.

\section{Funding}

BioMérieux is the sole private sponsor of the Global Point Prevalence Survey. The Global-PPS is also funded by a personal Methusalem grant to Herman Goossens of the Flemish government. BioMérieux has no role in study design, data collection, data analysis, data interpretation, or writing the report. Data are strictly confidential and stored anonymous at the coordinating center of the University of Antwerp.

\section{Availability of data and materials}

The datasets used and/or analysed during the current study are available from the corresponding author on reasonable request.

\section{Ethics approval and consent to participate}

All participating hospitals had to ask their local ethics committee for approval of the study protocol. A data-privacy excerpt for the conduction of the Global-PPS was made available for that purpose. All hospital data were coded with a unique number.

\section{Consent for publication}

Not applicable.

\section{Competing interests}

The authors declare that they have no competing interests.

\section{Author details}

${ }^{1}$ Healthcare-associated infections and antimicrobial resistance, Sciensano, Rue Juliette Wytsmanstraat 14, 1050 Brussels, Belgium. ${ }^{2}$ Laboratory of Medical Microbiology, University of Antwerp, Antwerp, Belgium. ${ }^{3}$ Belgian Antibiotic Policy Coordination Commission (BAPCOC), Direction General Healthcare, Federal Public Service Health, Food Chain Safety and Environment, Brussels, Belgium. ${ }^{4}$ Department of Microbiology, University of Hasselt, Hasselt, Belgium. ${ }^{5}$ Faculty of Medicine, Université Libre de Bruxelles (ULB), Brussels, Belgium.

Received: 18 October 2019 Accepted: 12 December 2019

Published online: 13 January 2020

\section{References}

1. Humphreys H, Smyth ET. Prevalence surveys of healthcare-associated infections: what do they tell us, if anything? Clin Microbiol Infect. 2006;12(1):2-4.

2. Gordts B, Vrijens F, Hulstaert F, Devriese S, Van de Sande S. The 2007 Belgian national prevalence survey for hospital-acquired infections. J Hosp Infect. 2010;75(3):163-7.

3. European Centre for Disease Prevention and Control (ECDC). Point prevalence survey of healthcare-associated infections and antimicrobial use in European acute care hospitals: surveillance report 2011-2012. Stockholm: ECDC; 2013.

4. Versporten A, Zarb P, Caniaux I, Gros MF, Drapier N, Miller M, et al. Antimicrobial consumption and resistance in adult hospital inpatients in 53 countries: results of an internet-based global point prevalence survey. Lancet Glob Health. 2018;6(6):e619-29.

5. Versporten A, Magerman K, Drapier N, Goossens H. The Global Point Prevalence Survey of antimicrobial consumption and resistance (Global-PPS) in 100 Belgian hospitals. Abstract ECCMID. Amsterdam; 2016. Poster NEV0684. http://www.global-pps.com/wp-content/uploads/ECCMID-2016_ Belgium.pdf. Accessed 18 Oct 2019

6. Federal Public Service health, food chain safety and environment - Dienst Datamanagement -Directoraat-Generaal Gezondheidszorg. List of Belgian hospitals. Version 62017.

7. European Centre for Disease Prevention and Control (ECDC). Point prevalence survey of healthcare associated infections and antimicrobial use in European acute care hospitals. Protocol version 5.3. Stockholm: ECDC; 2016.

8. World Health Organization (WHO) Collaborating Centre for Drugs Statistics Methodology. DDD and ATC-classification, 2019. https://www.whocc.no/ atc_ddd_index/. Accessed 18 Oct 2019.

9. Plachouras D, Karki T, Hansen S, Hopkins S, Lyytikainen O, Moro ML, et al. Antimicrobial use in European acute care hospitals: results from the second point prevalence survey (PPS) of healthcare-associated infections and antimicrobial use, 2016 to 2017. Euro Surveill. 2018;23(46):1800393. https:// doi.org/10.2807/1560-7917.ES.23.46.1800393.

10. Catry B, Mertens K, Latour K, Legiest B, Duysburgh E, Papadopoulos T, et al. Care Infections \& Antibiotic Resistance in Belgium. J Epidemiol Public Health Rev. 2018;3(2). https://doi.org/10.16966/2471-8211.165.

11. Belgian Hospitals - Surveillance of Antimicrobial Consumption (BeH-SAC). National reports on Healthstat, 2016. Available from: https://www.healthstat. be/. Accessed 18 Oct 2019

12. Belgian Antibiotic Policy Coordination Committeee (BAPCOC): Beleidsnota legislatuur 2014-2019. https://overlegorganen.gezondheid.belgie.be/sites/ default/files/documents/belgische_commissie_voor_de_coordinatie_van het_antibioticabeleid/19100224.pdf. Accessed 18 Oct 2019.

13. Pollack LA, Plachouras D, Sinkowitz-Cochran R, Gruhler H, Monnet DL, Weber JT, et al. A concise set of structure and process indicators to assess and compare antimicrobial stewardship programs among EU and US hospitals: results from a multinational expert panel. Infect Control Hosp Epidemiol. 2016;37(10):1201-11

14. Suetens C, Latour K, Karki T, Ricchizzi E, Kinross P, Moro ML, et al. Prevalence of healthcare-associated infections, estimated incidence and composite antimicrobial resistance index in acute care hospitals and long-term care facilities: results from two European point prevalence surveys, 2016 to 2017. Euro Surveill. 2018; 23(46):1800516. https://doi.org/10.2807/1560-7917.ES. 2018.23.46.1800516. 
15. Cairns S, Gibbons C, Milne A, King H, Llano M, MacDonald L, et al. Results from the third Scottish National Prevalence Survey: is a population health approach now needed to prevent healthcare-associated infections? J Hosp Infect. 2018;99(3):312-7.

16. Duysburgh E, Dequeker S, Mortgat L. Kwaliteitsindicatoren voor ziekenhuishygiëne in acute ziekenhuizen. Jaarrapport 2017. http://www.nsih. be/download/IQ/Rapport_Ql_2017_NL.pdf. Accessed 18 Oct 2019.

17. Annemans $L$, Boeckxstaens $P$, Borgermans $L$, De Smedt D, Duchesnes $C$, Heyrman J, et al. Advantages, disadvantages and feasibility of the introduction of 'Pay for Quality' programmes in Belgium. Health Services Research (HSR). Brussels: Belgian Health Care Knowledge Centre (KCE); 2009. KCE Reports 118C. D/2009/10.273/52

\section{Publisher's Note}

Springer Nature remains neutral with regard to jurisdictional claims in published maps and institutional affiliations.

Ready to submit your research? Choose BMC and benefit from:

- fast, convenient online submission

- thorough peer review by experienced researchers in your field

- rapid publication on acceptance

- support for research data, including large and complex data types

- gold Open Access which fosters wider collaboration and increased citations

- maximum visibility for your research: over $100 \mathrm{M}$ website views per year

At BMC, research is always in progress.

Learn more biomedcentral.com/submissions 